\title{
LOVE AND LIBERATION
}





\section{LOVE AND \\ LIBERATION}

Humanitarian Work in Ethiopia's Somali Region

\section{Lauren Carruth}




\section{Copyright () 2021 by Cornell University}

All rights reserved. Except for brief quotations in a review, this book, or parts thereof, must not be reproduced in any form without permission in writing from the publisher. For information, address Cornell University Press, Sage House, 512 East State Street, Ithaca, New York 14850. Visit our website at cornellpress.cornell.edu.

First published 2021 by Cornell University Press

Library of Congress Cataloging-in-Publication Data

Names: Carruth, Lauren, 1980- author.

Title: Love and liberation : humanitarian work in Ethiopia's Somali region / Lauren Carruth.

Description: Ithaca [New York] : Cornell University Press, 2021.|

Includes bibliographical references and index.

Identifiers: LCCN 2021002961 (print) | LCCN 2021002962 (ebook) |

ISBN 9781501759475 (hardcover) | ISBN 9781501759666 (paperback) |

ISBN 9781501759482 (pdf) | ISBN 9781501759499 (epub)

Subjects: LCSH: Humanitarian assistance-Ethiopia—Somali Region. |

Humanitarianism-Political aspects-Ethiopia—Somali Region. |

Humanitarian aid workers-Ethiopia-Somali Region.

Classification: LCC HV593.A35 C37 2021 (print) | LCC HV593.A35 (ebook) |

DDC 361.2/609632-dc23

LC record available at https://lccn.loc.gov/2021002961

LC ebook record available at https://lccn.loc.gov/2021002962

Cover image: Ebony Iman Dallas, Golden Hearts from the Horn, Through Abahay's Eyes Series. Acrylic, golden leafing, Kenyan textiles, $15 \times 20$ in. Used by permission of the artist. 